\title{
THE COHOMOLOGY RING OF PRODUCT COMPLEXES $\left({ }^{1}\right)$
}

\author{
BY \\ F. P. PALERMO
}

Introduction. The Künneth formula enables one to determine the integral cohomology group of the product $X \times Y$ of the spaces $X$ and $Y$ in terms of the integral cohomology groups $H(X)$ and $H(Y)$. However, this formula does not enable one to determine the multiplicative structure of the cohomology ring $H(X \times Y)$ in terms of the integral cohomology rings $\left({ }^{2}\right) H(X)$ and $H(Y)$. It is natural to ask the question: Is the integral cohomology ring $H(X \times Y)$ determined by the integral cohomology rings $H(X)$ and $H(Y)$ ?

This question is answered in the negative by the following example:

Let $X_{1}=Y_{1}$ be the union of the real projective plane and a one-sphere (circle) with one point in common. Let $X_{2}=Y_{2}$ be a Klein bottle. It is easy to check that the rings $H\left(X_{1}\right)$ and $H\left(X_{2}\right)$ are isomorphic. However, the rings $H\left(X_{1} \times Y_{1}\right)$ and $I I\left(X_{2} \times Y_{2}\right)$ are not isomorphic. In particular, there is a onedimensional cohomology class and a three-dimensional cohomology class in $I I\left(X_{2} \times Y_{2}\right)$ whose product is a nonzero four-dimensional cohomology class. On the other hand, all products of one-dimensional and three-dimensional cohomology classes of $H\left(X_{1} \times Y_{1}\right)$ are zero. Hence these two cohomology rings cannot be isomorphic. These assertions will follow readily from the theorems proved below.

Since the answer to the above question is negative, it is natural to inquire: What information about the cohomology rings of $X$ and $Y$ is needed to determine the integral cohomology ring of $X \times Y$ ? Let $H(X, n)$ denote the cohomology ring of $X$ with the integers modulo $n$ as coefficients. Following J. H. C. Whitehead [8], and Bockstein [2], we define the spectrum of coho. mology rings of $X$, or simply the cohomology spectrum of $X$, to be the set of cohomology rings $I I(X, n), n \geqq 0$, together with the coefficient homomorphisms: $I(X, n) \rightarrow I(X, m), m>0$ and the Bockstein homomorphisms of degree $+1: H(X, m) \rightarrow H(X, 0), m>0$. (These homomorphisms are defined in section (1).) Bockstein [2] stated but did not prove that the cohomology spectra of $X$ and $Y$ determine the cohomology ring $H(X \times Y)$. From these spectra he constructed a group isomorphic to the group $H(X \times Y)$, however, he did not introduce any products in this construction; thus the question of

Presented to the Society, under the title The cohomology rings of product spaces on December 27, 1954; received by the editors March 11, 1956 and, in revised form, July 19. 1956.

(1) A condensed version of a doctoral dissertation submitted to Brown University. The author wishes to thank Professor W. S. Massey for his advice and guidance in the writing of this thesis.

(2) The multiplication in the cohomology ring is the "cup" product. The cohomology ring is a graded, skew-commutative, associative ring. 
the determination of the integral cohomology ring of $X \times Y$ was left open. Bockstein also gave a construction for determining the groups $H(X \times Y, n)$, the coefficient homomorphisms and the Bockstein homomorphisms.

In this paper, the problem of determining the cohomology ring of the product $X \times Y$ is treated in a purely algebraic fashion. We start with a cochain complex $K$, and study the cohomology spectrum of $K$. Various relations which exist within this spectrum are indicated in the first chapter of this paper. The tensor product $K \otimes L$ of two cochain complexes $K$ and $L$ corresponds to the cartesian product $X \times Y$ of the topological spaces $X$ and $Y$. Thus, the algebraic problem amounts to the determination of the cohomology ring $H(K \otimes L)$. In the second chapter, Bockstein's construction is used to construct a group $R_{0}(K, L)$, which is isomorphic to the group $H(K \otimes L)$, if the complexes $K$ and $L$ are finitely generated. Products are introduced in $R_{0}(K, L)$, giving a ring which is proved to be isomorphic to the cohomology ring $H(K \otimes L)$. It is seen that only a finite collection of rings of the spectra of $K$ and $L$ are actually needed in the determination of the ring $H(K \otimes L)$ when $K$ and $L$ are finitely generated. Thus, this construction lends itself to computation.

In Chapter III, it is shown that the cohomology ring $H(K \otimes L, n)$ is determined by the spectra of $K$ and $L$. The coefficient and Bockstein homomorphisms for $K \otimes L$ are considered in Chapter IV, and the various constructions are shown to commute with direct limits. Thus the result can be extended to infinite complexes and Cech cohomology.

\section{Chapter I. The Cohomology spectrum of a cochain complex}

1. Definitions and conventions. A pair $(C(K), \delta)$ is called a graded differential group if $C(K)$ is the direct sum of subgroups $C^{r}(K)$, where $C^{r}(K)=0$ for $r<0$, and $\delta$ is a differential operator homogeneous of degree +1 (or -1 ) i.e. $\delta$ is an endomorphism of $K$ with $\delta C^{r}(K) \subset C^{r+1}(K)$ and $\delta \delta=0$. An automorphism $\omega$ of $C(K)$ is said to be an involution of $C(K)$ if $\omega \delta+\delta \omega=0$ and $\omega \omega(x)=x$ for all $x \in C(K)$. Usually $\omega$ will be the involution defined by $\omega(x)$ $=(-1)^{p} x$ for $x \in C^{p}(K)$.

A triple $(C(K), \delta, \omega)$ is called a graded differential ring if $(C(K), \delta)$ is a graded differential group, $\omega$ is an involution, $C(K)$ has an identity $1 \in C^{0}(K)$, $x \cdot y \in C^{p+u}(K)$ for $x \in C^{p}(K)$ and $y \in C^{u}(K)$, and $\delta(x y)=(\delta x) y+\omega(x) \delta(y)$.

$K=(C(K), \delta, \omega)$ will be called a cochain complex if $(C(K), \delta, \omega)$ is a graded differential ring with $\delta$ homogeneous of degree +1 .

We let $H(K, n)$ denote the cohomology ring of the cochain complex $K \otimes Z_{n}=\left(C(K) \otimes Z_{n}, \delta \otimes 1, \omega \otimes 1\right)$, i.e. $H(K, n)=$ kernel $(\delta \otimes 1)$ modulo image $\delta \otimes 1$ where 1 is the identity on $Z_{n}$, the integers modulo $n$. If $n=0, H(K, 0)$, shortened to $H(K)$, denotes the integral cohomology ring of $K$, i.e. kernel $\delta$ modulo image $\delta$. The involution $\omega$ of $K$ induces an involution $\omega$ on each $H(K, n)$. We let $(x)_{n}$ denote the cohomology class in $H(K, n)$ of a cochain 
$x$ which is a cocycle modulo $n$, i.e. $\delta x=n y$ for some cochain $y$ in $K$.

For each pair of integers $m \geqq 0, n>0$, the coefficient homomorphisms $h_{n, m}: H(K, m) \rightarrow H(K, n)$ are defined by the formula

$$
h_{n, m}\left(x_{m}\right)=\left(\frac{n}{(n, m)} x\right)_{n}
$$

for any cochain $x$ which is a cocycle modulo $m$, where $(m, n)$ denotes the greatest common divisor of $m$ and $n$. It is easy to check that $h_{n, m}$ is well defined, and is a group homomorphism which is homogeneous of degree zero.

For $n>0$, the Bockstein homomorphism $\Delta_{n}: H(K, n) \rightarrow H(K)$ is defined by the formula

$$
\Delta_{n}\left(x_{n}\right)=\left(\frac{1}{n} \delta x\right)_{0} .
$$

It is easy to check that $\Delta_{n}$ is a well defined group homomorphism which is homogeneous of degree +1 .

2. The cohomology spectrum of a cochain complex. The cohomology spectrum of a cochain complex is defined to be the collection of cohomology rings $H(K, n)$ together with the Bockstein and coefficient homomorphisms.

We shall now list some relations which exist between the Bockstein and coefficient homomorphisms of the cohomology spectrum of the cochain complex $K$. Their proofs are consequences of the definitions. Most of these formulae, together with their proofs, are in J. H. C. Whitehead [8].

The following two conditions are satisfied by the Bockstein and coefficient homomorphisms:

$$
\begin{aligned}
h_{k, m} h_{m, n} & =\frac{m(n, k)}{(m, k)(m, n)} h_{k, n}, \\
\Delta_{m} h_{m, n} & =\frac{n}{(m, n)} \Delta_{n} .
\end{aligned}
$$

Let $\sum(K)$ denote the direct sum of the rings $H(K, n)$ for all $n \geqq 0$. A product is introduced in $\sum(K)$ by the formula

$$
x y=h_{c, m}(x) h_{c, n}(y)
$$

where $x$ is in $H(K, m), y$ is in $H(K, n)$ and $c=(m, n)$. This product is extended to all of $\sum(K)$ by linearity. It is easy to check that $\sum(K)$ is an associative skew commutative ring with this product.

The Bockstein and coefficient homomorphisms satisfy the following relations with respect to these products:

$$
h_{k, m}(x) h_{k, n}(y)=\frac{k(k, m, n)}{(k, m)(k, n)} h_{k, c}(x y),
$$




$$
\Delta_{c, c}(x y)=\left[\Delta_{c, c} h_{c, m}(x)\right] y+\omega(x)\left[\Delta_{c, c} h_{c, n}(y)\right],
$$

where $x \in H(K, m), y \in H(K, n), c=(m, n)$ and $\Delta_{c, c}=h_{c, 0} \Delta_{c}$. If $k \mid n$ and $m=n$, formula (2.4) reduces to

$$
h_{k, m}(x) h_{k, m}(y)=h_{k, m}(x y)
$$

i.e. $h_{k, m}: H(K, m) \rightarrow H(K, k)$ is a ring homomorphism if $k$ divides $m$.

The following two relations have some important consequences:

$$
\begin{aligned}
& \Delta_{n}\left(y \cdot h_{n, 0}(x)\right)=\Delta_{n}(y) \cdot x, \\
& \Delta_{n}\left(h_{n, 0}(x) \cdot y\right)=\omega(x) \cdot \Delta_{n}(y)
\end{aligned}
$$

for $x \in H(K)$ and $y \in H(K, n)$. If in formulae (2.7) and (2.8) $x=\Delta_{n}(z)$ for some $z$ in $H(K, n)$, these equations take the form

$$
\begin{aligned}
& \Delta_{n}\left(y \cdot \Delta_{n, n}(z)\right)=\Delta_{n}(y) \cdot \Delta_{n}(z), \\
& \Delta_{n}\left(\Delta_{n, n}(z) \cdot y\right)=\omega\left(\Delta_{n}(z)\right) \cdot \Delta_{n}(y) .
\end{aligned}
$$

These last two formulae suggest that $\Delta_{n}: H(K, n) \rightarrow H(K)$ will become a ring homomorphism if a new product * is introduced in $H(K, n)$ by either one of the formulae

$$
\begin{aligned}
& x * y=x \cdot \Delta_{n, n}(y), \\
& x * y=\omega \Delta_{n, n}(x) \cdot y
\end{aligned}
$$

for $x$ and $y$ in $H(K, n)$.

Let $K$ and $K^{\prime}$ be cochain complexes and suppose $F: K \rightarrow K^{\prime}$ is an allowable cochain map, i.e., $F$ satisfies the following conditions:

$$
\begin{aligned}
\text { (i) } F \delta & =\delta F, \\
\text { (ii) } F \omega & =\omega F .
\end{aligned}
$$

Then $F$ induces homomorphisms $F_{n}: H(K, n) \rightarrow H\left(K^{\prime}, n\right)$ for each $n$. These induced homomorphisms are related to the Bockstein and coefficient homomorphisms and the multiplication by the formulae

$$
\begin{aligned}
& \text { (a) } F_{n} h_{n, m}=h_{n, m} F_{m}, \\
& \text { (b) } F_{0} \Delta_{n}=\Delta_{n} F_{n}, \\
& \text { (c) } F_{n}(x y)=F_{n}\left(x_{i}\right) F_{n}(y),
\end{aligned}
$$

where $x$ and $y$ are in $H(K, n)$.

The Bockstein and coefficient homomorphisms preserve direct sums. Stated precisely, we have the direct sum theorem:

TheOREM 2.1. If $K=\sum_{i} K_{i}$ (direct sum) where each $K_{i}$ is an allowable subcomplex (i.e. $\delta K_{i} \subset K_{i}$ ) of the cochain complex $K$, then

$$
\text { (a) } H(K, n)=\sum_{i} H\left(K_{i}, n\right) \text { for each } n \text {. (Direct sum.) }
$$


(b) $h_{m, n}\left(H\left(K_{i}, n\right)\right) \subset H\left(K_{i}, m\right)$.

(c) $\Delta_{n}\left(H\left(K_{i}, n\right)\right) \subset H\left(K_{i}\right)$.

Chapter II. The integral cohomology Ring $H(K \otimes L)$

3. The group $H(K \otimes L)$. Let $K$ and $L$ be cochain complexes. The tensor product $K \otimes L$ of the cochain complexes $K$ and $L$ is defined to be the graded differential ring $(C(K) \otimes C(L),(\delta \otimes 1)+(\omega \otimes \delta), \omega \otimes \omega)$ with multiplication defined by the formula

$$
(x \otimes y) \cdot(u \otimes v)=(-1)^{p q} x u \otimes y v
$$

for $y \in C^{p}(K)$ and $u \in C^{q}(L)$. Let $S_{0}(K, L)$ be the weak direct sum of the groups $H(K, n) \otimes H(L, n)$ for all non-negative integers $n$. Define $\phi_{0}: S_{0}(K, L)$ $\rightarrow H(K \otimes L)$ as follows:

$$
\begin{aligned}
\phi_{0} \mid H(K) \otimes H(L) & =\alpha_{0}, \\
\phi_{0} \mid H(K, n) \otimes H(L, n) & =\Delta_{n} \alpha_{n}
\end{aligned}
$$

where $\alpha_{n}: H(K, n) \otimes H(L, n) \rightarrow H(K \otimes L, n)$ is the homomorphism induced by the bilinear map of $H(K, n) \times H(L, n) \rightarrow H(K \otimes L, n)$ which carries $\left(x_{n}, y_{n}\right)$ into $(x \otimes y)_{n}$. Thus we have $\alpha_{n}\left(x_{n} \otimes y_{n}\right)=(x \otimes y)_{n}$.

THEOREM 3.1. If $K$ and $L$ are finitely generated free cochain complexes, then

(a) $\phi_{0}$ is a homomorphism onto,

(b) kernel $\phi_{0}$ is the subgroup $Q_{0}(K, L)$ of $S_{0}(K, L)$ generated by the following relators:

(i) $h_{i, j}\left(x_{j}\right) \otimes y_{i}-x_{j} \otimes h_{j, i}\left(y_{i}\right)$, $i \mid j$ or $j \mid i$,

(ii) $\Delta_{i}\left(x_{i}\right) \otimes y_{0}-x_{i} \otimes h_{i, 0}\left(y_{0}\right)$, $i>0$,

(iii) $x_{0} \otimes \Delta_{i}\left(y_{i}\right)-h_{i, 0}\left(\omega\left(x_{0}\right)\right) \otimes y_{i}$, $i>0$.

Proof. A finitely generated free cochain complex is the direct sum of elementary allowable subcomplexes, an elementary complex being a complex $E$ of one of the following two types:

(1) $E$ has one free generator $c$ with $\delta c=0$,

(2) $E$ has two free generators $a$ and $b$ with $\delta a=t b$ and $\delta b=0$ where $t$ is an integer greater than 1 .

Since $K$ and $L$ are finitely generated free cochain complexes, they each split in to the direct sum of elementary complexes. Hence it suffices to prove (a) and (b) for the case where $K$ and $L$ are elementary complexes $\left({ }^{3}\right)$ because of the following direct sum lemma.

Lemma 3.2. If $K=\sum_{i} K_{i}, L=\sum_{j} L_{j}$ where $K_{i}$ and $L_{j}$ are allowable subcomplexes of $K$ and $L$ respectively, then

(3) Some details of such a proof are given in the appendix. 


$$
\begin{aligned}
& \text { (i) } S_{0}(K, L)=\sum_{i, j} S_{0}\left(K_{i}, L_{j}\right), \\
& \text { (ii) } \phi_{0}\left(S_{0}\left(K_{i}, L_{j}\right)\right) \subset H\left(K_{i} \otimes L_{j}\right) .
\end{aligned}
$$

The proof of this lemma is a straightforward verification using the facts that homology and tensor products preserve direct sums (Eilenberg and Steenrod [5, p. 141]).

4. The multiplication in $H(K \otimes L)$. Thus far, the ring structure of $H(K \otimes L)$ has not been considered. The multiplication in $K \otimes L$ is given by formula (3.1). In this section products will be introduced in $S_{0}(K, L)$. It is convenient to write $h_{i, j}^{\prime}$ in place of $h_{i, j} \otimes h_{i, j}: H(K, j) \otimes H(L, j) \rightarrow H(K, i) \otimes H(L, i)$.

Since $H(K, n)$ and $H(L, n)$ are graded rings, $H(K, n) \otimes H(L, n)$ is given a ring structure in the standard way, i.e. for $x$ in $H^{p}(K, n), y$ in $H^{q}(L, n), u$ in $H(K, n)$ and $v$ in $H(L, n)$

$$
(u \otimes y) \cdot(x \otimes v)=(-1)^{p q}(u x) \otimes(y v) .
$$

With this multiplication, $H(K, n) \otimes H(L, n)$ is an associative ring. This multiplication is extended to $S_{0}(K, L)=\sum_{n \geqq 0} H(K, n) \otimes H(L, n)$ by the formula

$$
x \cdot y=h_{c, i}^{\prime}(x) \cdot h_{c, j}^{\prime}(y)
$$

where $x$ is in $H(K, i) \otimes H(L, i), y$ is in $H(K, j) \otimes H(L, j)$ and $c=(i, j)$. With this multiplication, $S_{0}(K, L)$ is an associative ring.

In $\$ 2$, a multiplication was introduced in the direct sum of the cohomology rings $H(K, i), i \geqq 0$. We list some properties of the homomorphisms $\alpha_{j}$ : $H(K, j) \otimes H(L, j) \rightarrow H(K \otimes L, j)$ and the coefficient homomorphisms with respect to these products.

$$
\begin{array}{rlr}
\alpha_{i} h_{i, j}^{\prime} & =\frac{i}{(i, j)} h_{i, j} \alpha_{j}, & i>0, j \geqq 0, \\
\alpha_{i}(x y) & =\alpha_{i}(x) \alpha_{i}(y) \text { for } x \text { and } y \text { in } H(K, i) \otimes H(L, i) . \\
\alpha_{i} h_{i, j}^{\prime} & =h_{i, j} \alpha_{j} \text { if } i \mid j, \\
\alpha_{c}(x y) & =\alpha_{i}(x) \cdot \alpha_{j}(y) \text { for } x \text { in } H(K, i) \otimes H(L, i), \\
& y \text { in } H(K, j) \otimes H(L, j) \text { and } c=(i, j) .
\end{array}
$$

Formulae (4.3) and (4.4) follow immediately from the definitions while formulae (4.5) and (4.6) are consequences of (4.3) and (4.4).

However, $\phi_{0}: S_{0}(K, L) \rightarrow H(K \otimes L)$ does not preserve these products. This can be seen just from considerations of degrees. We noted in $\$ 2$ that a new multiplication * can be introduced in $H(K, n)$ so that $\Delta_{n}: H(K, n) \rightarrow H(K)$ becomes a ring homomorphism. Following this lead, we introduce a coboundary endomorphism $D_{n}$ in $H(K, n) \otimes H(L, n)$ for $n>0$ by the formula

$$
D_{n}=\Delta_{n, n} \otimes I+\omega \otimes \Delta_{n, n}
$$


where $I$ is the identity on $H(L, n)$ and $\omega$ is the involution of $H(K, n)$. An involution $\omega^{\prime}$ of $H(K, n) \otimes H(L, n)$ is defined by $\omega^{\prime}=\omega \otimes \omega$.

The following properties of $D_{n}$ and $\omega^{\prime}$ are easily verified using formulae (4.3), (4.4), (4.5) and (4.6).

$$
\begin{aligned}
& D_{n} D_{n}=0, \quad D_{n} \omega^{\prime}+\omega^{\prime} D_{n}=0 . \\
& \alpha_{n} D_{n}=\Delta_{n, n} \alpha_{n}, \quad \alpha_{n} \omega^{\prime}=\omega \alpha_{n} . \\
& \text { If } c \mid i, \quad D_{c} h_{c, i}^{\prime}=\frac{i}{c} h_{c, i}^{\prime} D_{i} . \\
& \Delta_{n} \alpha_{n}\left(x \cdot D_{n}(y)\right)=\Delta_{n} \alpha_{n}(x) \Delta_{n} \alpha_{n}(y)=\Delta_{n} \alpha_{n}\left(\omega^{\prime} D_{n}(x) \cdot y\right),
\end{aligned}
$$

where $x$ and $y$ are in $H(K, n) \otimes H(L, n)$.

A multiplication * is defined in $S_{0}(K, L)$ as follows: For each pair of positive integers $i$ and $j$, choose integers $\alpha$ and $\beta$ so that $\alpha i+\beta j=(i, j)$. For $x$ in $H(K, i) \otimes H(L, i), y$ in $H(K, j) \otimes H(L, j)$, we define

$$
\begin{aligned}
x * y & =x y \text { if } j=0 \\
& =\omega^{\prime}(x) y \text { if } j>0, i=0 \\
& =\alpha x \cdot D_{j}(y)+\beta \omega^{\prime}\left(D_{i}(x)\right) \cdot y \text { if } i>0, j>0 .
\end{aligned}
$$

This product is extended to $S_{0}(K, L)$ by linearity. $S_{0}(K, L)$ with this multiplication is a (nonassociative) ring.

The following theorem shows the importance of the * products.

THEOREM 4.1. $\phi_{0}: S_{0}(K, L) \rightarrow H(K \otimes L)$ is a ring homomorphism with respect to the * products in $S_{0}(K, L)$; i.e. $\phi_{0}(x * y)=\phi_{0}(x) \cdot \phi_{0}(\dot{y})$.

Proof. It suffices to check this for

$$
x \text { in } H(K, i) \otimes H(L, i) \text { and } y \text { in } H(K, j) \otimes H(L, j) .
$$

There are four cases to be considered. Case $1, i=j=0$ follows from formula (4.4); Case $2, i>0, j=0$; and Case $3, i=0, j>0$ follow from formulae (4.6), (2.6) and (2.8). Case $4, i>0, j>0$ follows from formulae (4.13) and (4.14), below, which are consequences of formulae (4.9), (4.10) and (4.11).

For $x$ in $H(K, i) \otimes H(L, i)$ and $y$ in $H(K, j) \otimes H(L, j), i>0, j>0, c=(i, j)$,

$$
\begin{aligned}
\Delta_{c} \alpha_{c}\left(x D_{j}(y)\right) & =\frac{i}{c} \Delta_{i} \alpha_{i}(x) \Delta_{j} \alpha_{j}(y), \\
\Delta_{c} \alpha_{c}\left(\omega^{\prime}\left(D_{i}(x)\right) y\right) & =\frac{j}{c} \Delta_{i} \alpha_{i}(x) \Delta_{j} \alpha_{j}(y) .
\end{aligned}
$$

We should observe that in this theorem we have not assumed $K$ and $L$ to be finitely generated cochain complexes.

Combining Theorems 3.1 and 4.1 , we obtain 
COROLLARY 4.2. If $K$ and $L$ are finitely generated free cochain complexes, then $Q_{0}(K, L)$ is an ideal of $S_{0}(K, L)$ with * as multiplication.

Thus we have the very important

Corollary 4.3. $\phi_{0}$ induces a ring isomorphism $\phi_{0}^{*}$ of $S_{0}(K, L)$ modulo $Q_{0}(K, L)$ onto $H(K \otimes L)$ when $K$ and $L$ are finitely generated and free.

The multiplication * introduced in $S_{0}(K, L)$ depended on choices of integers $\alpha$ and $\beta$. Theorem 4.1 shows that this multiplication is determined modulo kernel $\phi_{0}$. Thus if $K$ and $L$ are finitely generated and free, $*$ is determined modulo $Q_{0}(K, L)$. However, it can be shown directly that if a second multiplication ${ }^{*}$ ' is introduced in $S_{0}(K, L)$ using a different choice of integers, then for $x$ in $H(K, i) \otimes H(L, i), y$ in $H(K, j) \otimes H(L, j)$ and $c=(i, j)$

$$
x * y-x *^{\prime} y=D_{c}\left(k \omega^{\prime}(x) y\right) \text { for some integer } k \text {. }
$$

But $D_{c}(u)$ is easily seen to be in $Q_{0}(K, L)$. Thus $*$ is unique modulo $Q_{0}(K, L)$.

Suppose $K$ and $L$ are finitely generated free cochain complexes. Let $T$ be the collection of all integers $n$ such that $n=0$ or $n=(t, s)$ where $t$ ranges over the torsion coefficients of $K$ and $s$ ranges over the torsion coefficients of $L$ (Eilenberg and Steenrod [5, p. 138]). If we examine the proof that $\phi_{0}$ is onto $H(K \otimes L)$, we see that only the groups $H(K, n) \otimes H(L, n)$ for $n$ in $T$ are needed. However $\sum_{n \in T} H(K, n) \otimes H(L, n)$ need not be a subring of $S_{0}(K, L)$. Let $T^{\prime}$ be the smallest collection of integers which contains $T$ and is closed with respect to taking greatest common divisors. Then clearly

$$
\sum_{n \in T^{\prime}} H(K, n) \otimes H(L, n) .
$$

is a subring of $S_{0}(K, L)$ which contains the subring generated by

$$
\sum_{n \in T} H(K, n) \otimes H(L, n) \text {. }
$$

Thus the cohomology ring $H(K \otimes L)$ is determined by the finite collection of cohomology rings $H(K, n)$ and $H(L, n)$ for $n$ in $T^{\prime}$ if $K$ and $L$ are finitely generated and free.

\section{Chapter III. The cohomology ring $H(K \otimes L, n)$}

5. The group $H(K \otimes L, n), n>0$. Suppose $K$ and $L$ are cochain complexes. Let $P_{n}(K, L)$ be the direct sum of the groups $H(K, i) \otimes H(L, i)$ for all integers $i$ which divide $n$. Let $S_{n}(K, L)$ be the group $\operatorname{direct}\left({ }^{4}\right)$ product

$$
P_{n}(K, L) \times P_{n}(K, L) \text {. }
$$

We define $\theta_{n}: P_{n}(K, L) \rightarrow H(K \otimes L, n)$ by the formula

$$
\theta_{n} \mid H(K, i) \otimes H(L, i)=h_{n, i} \alpha_{i}
$$

(4) We use the direct product notation here because both factors are the same, while for $P_{n}(K, L)$ we used the direct sum notation because all the factors were distinct. 
and $\phi_{n}: P_{n}(K, L) \times P_{n}(K, L) \rightarrow H(K \otimes L, n)$ by the formula

$$
\phi_{n}(x, y)=\theta_{n}(x)+\Delta_{n, n} \theta_{n}(y),
$$

for $x$ and $y$ in $P_{n}(K, L)$. Clearly, $\phi_{n}$ is a group homomorphism.

THEOREM 5.1. If $K$ and $L$ are finitely generated free cochain complexes, then

(a) $\phi_{n}$ is a homomorphism onto $H(K \otimes L, n)$.

(b) kernel $\phi_{n}$ is the subgroup $Q_{n}(K, L)$ of $S_{n}(K, L)$ generated by the following relators:

(i) $\left(0, h_{i, j}\left(x_{j}\right) \otimes y_{i}-x_{j} \otimes h_{j, i}\left(y_{i}\right)\right)$,

(ii) $\left(h_{i, j}\left(x_{j}\right) \otimes y_{i}-x_{j} \otimes h_{j, i}\left(y_{i}\right), 0\right)$,

(iii) $\left(D_{n}(u),-u\right)$,

where $i$ and $j$ both divide $n, x_{j} \in H(K, j), y_{i} \in H(L, i)$ and $u \in H(K, n) \otimes H(L, n)$.

As in Chapter II, it suffices to prove the theorem for $K$ and $L$ as elementary complexes $\left({ }^{5}\right)$ because of the following lemma.

Lemma 5.2. If $K=\sum_{i} K_{i}, L=\sum_{j} L_{j}$ where $K_{i}$ and $L_{j}$ are allowable subcomplexes of the cochain complexes $K$ and $L$ respectively, then

$$
\begin{aligned}
& S_{n}(K, L)=\sum_{i, j} S_{n}\left(K_{i}, L_{j}\right), \\
& \phi_{n}\left(S_{n}\left(K_{i}, L_{j}\right)\right) \subset H\left(K_{i} \otimes L_{j}, n\right) .
\end{aligned}
$$

The proof of this lemma is left to the reader.

Letting $R_{n}(K, L)=S_{n}(K, L) \bmod Q_{n}(K, L)$, we have

COROLLARY 5.3. If $K$ and $L$ are finitely generated free cochain complexes, $\phi_{n}: S_{n}(K, L) \rightarrow H(K \otimes L, n)$ induces an isomorphism $\phi_{n}^{*}: R_{n}(K, L) \rightarrow H(K \otimes L, n)$, $n>0$.

6. Products in $S_{n}(K, L)$. Thus far $S_{n}(K, L)$ has been given only a group structure as the direct product $P_{n}(K, L) \times P_{n}(K, L)$.

A multiplication will now be introduced in $S_{n}(K, L)$ by first introducing a multiplication in $P_{n}(K, L)=\sum_{i \mid n} H(K, i) \otimes H(L, i)$. It is easy to see that $P_{n}(K, L)$ is an ideal of $S_{0}(K, L)$ with respect to the skew multiplication defined by formulae (4.1) and (4.2), and also with respect to the * product defined by formula (4.12). Thus, in particular, $P_{n}(K, L)$ is closed with respect to both these multiplications. We introduce another product $\odot$ in $P_{n}(K, L)$ by defining for $x$ in $H(K, i) \otimes H(L, i)$ and $y$ in $H(K, j) \otimes H(L, j)$

$$
x \odot y=\frac{n}{[i, j]} x y
$$

${ }^{(5)}$ Details of such a proof are given in the appendix. 
where $[i, j]$ is the least common multiple of $i$ and $j$. This product is extended to all of $P_{n}(K, L)$ by linearity.

The following formulae show the relations between the homomorphism $\theta_{n}$ and the products defined on $P_{n}(K, L)$. These formulae are established using formulae (2.4), (2.5) and the definition of $\theta_{n}$.

$$
\begin{aligned}
\theta_{n}(x \odot y) & =\theta_{n}(x) \theta_{n}(y) ; \\
\theta_{n}\left(x \cdot D_{j}(y)\right) & =\frac{i}{c} \theta_{n}(x) \cdot \Delta_{n, n} \theta_{n}(y) ; \\
\theta_{n}\left(D_{i}(x) \cdot y\right) & =\frac{j}{c}\left[\Delta_{n, n} \theta_{n}(x) \cdot \theta_{n}(y)\right] ; \\
\theta_{n}(x * y) & =\alpha \frac{i}{c} \theta_{n}(x) \cdot \Delta_{n, n}\left(\theta_{n}(y)\right)+\beta \frac{i}{c} \omega\left(\Delta_{n, n} \theta_{n}(x)\right) \cdot \theta_{n}(y) ; \\
\Delta_{n, n} \theta_{n} & =h_{n, 0} \phi_{0} \mid P_{n}(K, L) ;
\end{aligned}
$$

where $x \in H(K, i) \otimes H(L, i), y \in H(K, j) \otimes H(L, j), c=(i, j), \alpha$ and $\beta$ are integers such that $\alpha i+\beta j=c$, and $*$ is defined using these integers (formula (4.12)).

For each $i$ and $j$ which divides $n$, choose integers $\alpha$ and $\beta$ such that $\alpha i+\beta j=c=(i, j)$ and suppose that $*$ has been defined using these integers. Let $x \in H(K, i) \otimes H(L, i), y \in H(K, j) \otimes H(L, j)$. A multiplication denoted by $* *$ is introduced in $S_{n}(K, L)=P_{n}(K, L) \times P_{n}(K, L)$ by the formulae

(i) $(0, x) * *(0, y)=(0, x * y)$,

(ii) $(0, x) * *(y, 0)=\left(-\omega^{\prime}(x) * y, \alpha \frac{i}{c} x \odot y\right)$,

$$
\begin{aligned}
& \text { (iii) }(y, 0) * *(0, x)=\left(y * x, \alpha \frac{i}{c} \omega^{\prime}(y) \odot x\right), \\
& \text { (iv) }(y, 0) * *(x, 0)=(y \odot x, 0) .
\end{aligned}
$$

This product is extended to all of $S_{n}(K, L)$ by linearity. It is easy to check that $S_{n}(K, L)$ with this multiplication is a (nonassociative) ring.

THEOREM 6.1. $\phi_{n}: S_{n}(K, L) \rightarrow H(K \otimes L, n)$ is a ring homomorphism with respect to the $* *$ product defined by formula $(6.7)$, i.e. $\phi_{n}(u * * v)=\phi_{n}(u) \cdot \phi_{n}(v)$ for $u$ and $v$ in $S_{n}(K, L)$.

Proof. It suffices to prove this for the four cases given in formula (6.7). Cases (i) and (iv) are immediate consequences of formulae (6.2) and (6.6). The proofs for cases (ii) and (iii) are similar, hence we only give a detailed proof for Case (ii). Note the use of formulae (5.1), (6.5), (6.2) and (2.5). 


$$
\begin{aligned}
\phi_{n}((0, x) * *(y, 0))= & \phi_{n}\left(-\omega^{\prime}(x) * y, \alpha \frac{i}{c} x \odot y\right) \\
= & -\theta_{n}\left(\omega^{\prime}(x) * y\right)+\alpha \frac{i}{c} \Delta_{n, n} \theta_{n}(x \odot y) \\
= & -\left[\alpha \frac{i}{c} \theta_{n}\left(\omega^{\prime}(x)\right) \cdot \Delta_{n, n} \theta_{n}(y)\right. \\
& +\beta \frac{j}{c} \omega\left(\Delta_{n, n} \theta_{n}\left(\omega^{\prime}(x)\right) \cdot \theta_{n}(y)\right] \\
& +\alpha \frac{i}{c}\left[\Delta_{n, n}\left(\theta_{n}(x)\right) \cdot \theta_{n}(y)+\omega \theta_{n}(x) \cdot \Delta_{n, n} \theta_{n}(y)\right] \\
= & \beta \frac{j}{c} \Delta_{n, n} \theta_{n}(x) \cdot \theta_{n}(y)+\alpha \frac{i}{c} \Delta_{n, n} \theta_{n}(x) \cdot \theta_{n}(y) \\
= & \Delta_{n, n} \theta_{n}(x) \cdot \theta_{n}(y) \\
= & \phi_{n}(0, x) \cdot \phi_{n}(y, 0) .
\end{aligned}
$$

The following corollaries are immediate consequences of this theorem and Theorem 5.1.

Corollary 6.2. $Q_{n}(K, L)$ is an ideal of $S_{n}(K, L)$ with respect to the ** product if $K$ and $L$ are finitely generated free cochain complexes.

COROLLARY 6.3. If $K$ and $L$ are finitely generated free cochain complexes, then $S_{n}(K, L)$ is associative modulo $Q_{n}(K, L)$, i.e. $x * *(y * * z) \equiv(x * * y) * * z$ $\bmod Q_{n}$.

It can be proved directly that the multiplication defined in $S_{n}(K, L)$ is unique modulo $Q_{n}(K, L)$. However if $K$ and $L$ are finitely generated and free, this is an immediate consequence of Theorems 5.1 and 6.1.

Since $Q_{n}(K, L)$ is an ideal of $S_{n}(K, L)$ with respect to the $* *$ product when $K$ and $L$ are finitely generated and free, $R_{n}(K, L)$ is a ring. Thus we can state Theorem 5.1 in the stronger form:

THEOREM 6.4. If $K$ and $L$ are finitely generated free cochain complexes, then $\phi_{n}^{*}: R_{n}(K, L) \rightarrow H(K \otimes L, n)$ is a ring isomorphism.

We will now apply the foregoing theory to the example given in the intro duction. We let $Z_{n}[u]$ denote a cyclic group of order $n$ with generator $u$. Since the Klein bottle $K$ and the union of the real projective plane and a onesphere $\left(P_{2} \bigvee S_{1}\right)$ have only two as torsion number, we need consider only the cohomology rings with integers and integers modulo two for coefficients. The integral cohomology groups and the cohomology groups with integers modulo two are as follows: 


$$
\begin{aligned}
H^{0}(K) & =H^{0}\left(P^{2} \bigvee S_{1}\right)=Z\left[(e)_{0}\right], \\
H^{1}(K) & =H^{1}\left(P^{2} \bigvee S_{1}\right)=Z\left[(c)_{0}\right], \\
H^{2}(K) & =H^{2}\left(P^{2} \bigvee S_{1}\right)=Z_{2}\left[(b)_{0}\right], \\
H^{0}(K, 2) & =H^{0}\left(P^{2} \bigvee S_{1}, 2\right)=Z_{2}\left[(e)_{2}\right], \\
H^{1}(K, 2) & =H^{1}\left(P^{2} \bigvee S_{1}, 2\right)=Z_{2}\left[(c)_{2}\right]+Z_{2}\left[(a)_{2}\right], \\
H^{2}(K, 2) & =H^{2}\left(P^{2} \bigvee S_{1}, 2\right)=Z_{2}\left[(b)_{2}\right] .
\end{aligned}
$$

In each case, these generators of the respective groups are related by the Bockstein and coefficient homomorphisms as follows:

\begin{tabular}{|c|c|c|c|}
\hline & $(e)_{0}$ & $(c)_{0}$ & $(b)_{0}$ \\
\hline$(e)_{0}$ & $(e)_{0}$ & $(c)_{0}$ & $(b)_{0}$ \\
\hline$(c)_{0}$ & $(c)_{0}$ & 0 & 0 \\
\hline$(b)_{0}$ & $(b)_{0}$ & 0 & 0 \\
\hline
\end{tabular}

$$
\begin{aligned}
\Delta_{2}\left((a)_{2}\right) & =(b)_{0}, & \Delta_{2}\left((e)_{2}\right) & =\Delta_{2}\left((c)_{2}\right)=\Delta_{2}\left((b)_{2}\right)=0, \\
h_{2,0}\left((e)_{0}\right) & =(e)_{2}, & h_{2,0}\left((c)_{0}\right) & =(c)_{2} \text { and } h_{2,0}\left((b)_{0}\right)=(b)_{2} .
\end{aligned}
$$

The multiplication table for the cohomology rings of these spaces is determined by giving the products for these generators. With integer coefficients we have the same multiplication table for the both spaces. Thus we have the following table:

However, with integers modulo two we have different products as indicated in the following tables:

\begin{tabular}{|c|c|c|c|c|}
\cline { 2 - 4 } \multicolumn{1}{c|}{} & $(e)_{2}$ & $(c)_{2}$ & $(a)_{2}$ & $(b)_{2}$ \\
\hline$(e)_{2}$ & $(e)_{2}$ & $(c)_{2}$ & $(a)_{2}$ & $(b)_{2}$ \\
$(c)_{2}$ & $(c)_{2}$ & 0 & $(b)_{2}$ & 0 \\
$(a)_{2}$ & $(a)_{2}$ & $(b)_{2}$ & $(b)_{2}$ & 0 \\
$(b)_{2}$ & $(b)_{2}$ & 0 & 0 & 0 \\
\hline
\end{tabular}

Klein Bottle K

\begin{tabular}{|c|c|c|c|c|}
\cline { 2 - 4 } \multicolumn{1}{c|}{} & $(e)_{2}$ & $(c)_{2}$ & $(a)_{2}$ & $(b)_{2}$ \\
\hline$(e)_{2}$ & $(e)_{2}$ & $(c)_{2}$ & $(a)_{2}$ & $(b)_{2}$ \\
$(c)_{2}$ & $(c)_{2}$ & 0 & 0 & 0 \\
$(a)_{2}$ & $(a)_{2}$ & 0 & $(b)_{2}$ & 0 \\
$(b)_{2}$ & $(b)_{2}$ & 0 & 0 & 0 \\
\hline
\end{tabular}


It can be easily seen that the rings determined by the above multiplication tables are not isomorphic. If we compute the cohomology groups of $K \times K$ and $\left(P_{2} \bigvee S_{1}\right) \times\left(P_{2} \bigvee S_{1}\right)$ in dimensions one and three, we find the following generators:

$$
\begin{array}{ll}
(c \otimes e)_{0}, & (e \otimes c)_{0} \text { in dimension one, } \\
(c \otimes b)_{0}, & (b \otimes c)_{0}, \text { in dimension two }
\end{array}
$$

and

$$
\left(\frac{1}{2} \delta(a \otimes a)\right)_{0}=(b \otimes a-a \otimes b)_{0} \text { in dimension three. }
$$

\begin{tabular}{|c|c|c|}
\hline & $c \otimes e$ & $e \otimes c$ \\
\hline $\begin{array}{c}(c \otimes b)_{0} \\
(b \otimes c)_{0} \\
(b \otimes a-a \otimes b)_{0}\end{array}$ & $\begin{array}{c}0 \\
0 \\
(b \otimes b)_{0}\end{array}$ & $\begin{array}{c}0 \\
0 \\
(b \otimes b)_{0}\end{array}$ \\
\hline
\end{tabular}

This gives rise to the following multiplication tables:

\begin{tabular}{|c|c|c|}
\hline & $(c \otimes e)_{0}$ & $(e \otimes c)_{0}$ \\
\hline$(c \otimes b)_{0}$ & 0 & 0 \\
\hline$(b \otimes c)_{0}$ & 0 & 0 \\
\hline$(b \otimes a-a \otimes b)_{0}$ & 0 & 0 \\
\hline
\end{tabular}

$K \times K$

$$
\left(P_{2} \vee S_{1}\right) \times\left(P_{2} \vee S_{1}\right)
$$

This substantiates the assertion made in the introduction.

We now consider a second and more startling example which shows that we actually need to consider not only the abstract ring structures but also the coefficient and Bockstein homomorphisms. Let us take for the spaces, the lens spaces $L(5,1)$ and $L(5,2)$ (cf. Seifert and Threlfall [7, p. 210]). We let $L_{1}=L(5,1)$ and $L_{2}=L(5,2)$. Both of these spaces have only five torsion. Hence, we need consider only the cohomology rings with integers and integers modulo five for coefficients along with the corresponding coefficient and Bockstein homomorphisms. It is left to the reader to verify that these spaces have isomorphic cohomology rings with integers and integers mod $\mathbf{5}$ for coefficients. However, one finds that there is no isomorphism between these rings which commutes with the Bockstein and coefficient homomorphisms. Upon computing the integral cohomology rings of the spaces $L_{1} \times L_{1}$ and $L_{2} \times L_{2}$, using the above constructions, one finds that there can exist no isomorphism (ring) between these rings. 


\section{Chapter IV. The cohomology SPECTRUm of $K \otimes L$}

7. The Bockstein and coefficient homomorphisms in $S_{n}(K, L)$. Thus far we have constructed, for each non-negative integer $n$, a ring $S_{n}(K, L)$. In this section homomorphisms are defined within this collection of rings which will correspond to the Bockstein and coefficient homomorphisms. These new homomorphisms will be denoted by the same symbols.

For $m$ and $n$ positive integers we define $h_{m, n}: S_{n}(K, L) \rightarrow S_{m}(K, L)$ by the formula

$$
\begin{aligned}
& h_{m, n}(x, 0)=\frac{[m, n]}{[m, i]}\left(h_{c, i}^{\prime}(x), 0\right), \\
& h_{m, n}(0, x)=\frac{m}{(m, n)}\left(\alpha h_{c, i}^{\prime} D_{i}(x), \beta h_{c, i}^{\prime}(x)\right)
\end{aligned}
$$

where $x$ is in $H(K, i) \otimes H(L, i), i \mid n, c=(m, i)$ and the integers $\alpha$ and $\beta$ satisfy $\alpha m+\beta i=c$. We also define $h_{m, 0}: S_{0}(K, L) \rightarrow S_{m}(K, L)$ by the formula

$$
\begin{array}{rlr}
h_{m, 0}(x) & =\left(h_{m, 0}^{\prime}(x), 0\right) & i \\
& =\left(\alpha h_{c, i}^{\prime} D_{i}(x), \beta h_{c, i}^{\prime}(x)\right) & i>0
\end{array}
$$

for $x$ in $H(K, i) \otimes H(L, i), c=(m, i)$ where the integers $\alpha$ and $\beta$ satisfy $\alpha m+\beta i=c$.

The Bockstein homomorphism $\Delta_{n}: S_{n}(K, L) \rightarrow S_{0}(K, L), n>0$, is defined by the formula

$$
\Delta_{n}(x, y)=x
$$

for $(x, y)$ in $S_{n}(K, L)$. We also define an involution $\omega_{n}$ of $S_{n}(K, L)$ for $n>0$ by the formula

$$
\omega_{n}(x, y)=\left(\omega^{\prime}(x),-\omega^{\prime}(y)\right) \quad n>0 .
$$

An involution $\omega_{0}$ of $S_{0}(K, L)$ is defined for $x$ in $H(K, i) \otimes H(L, i)$ by the formula

$$
\begin{aligned}
\omega_{0}(x) & =-\omega^{\prime}(x) & & i>0, \\
& =\omega^{\prime}(x) & & i=0 .
\end{aligned}
$$

It can be shown directly that $h_{m, n}\left(Q_{n}(K, L)\right) \subset Q_{m}(K, L)$ and $\Delta_{n}\left(Q_{n}(K, L)\right)$ $\subset Q_{0}(K, L)$. However, such a direct proof is a long and tedious computation. The two inclusions are immediate corollaries of the following theorem when $K$ and $L$ are finitely generated and free.

THEOREM 7.1. The homomorphisms $h_{m, n}, \Delta_{n}$ and $\omega_{n}$ defined above commute with $\phi_{n}$. Thus we have the following formulae:

$$
\phi_{n} h_{n, m}=h_{n, m} \phi_{m} \quad n>0, m \geqq 0,
$$




$$
\begin{aligned}
\phi_{0} \Delta_{m} & =\Delta_{m} \phi_{m} \\
\phi_{n} \omega_{n} & =\omega \phi_{n}
\end{aligned}
$$$$
m>0,
$$$$
n \geqq 0 \text {. }
$$

The proofs of these formulae are easy verifications and are left to the reader.

As an immediate consequence we have then

CoROLlary 7.2. If $K$ and $L$ are finitely generated free cochain complexes,

(i) $h_{m, n}\left(Q_{n}(K, L)\right) \subset Q_{m}(K, L)$ $m>0, n \geqq 0 ;$

(ii) $\Delta_{n}\left(Q_{n}(K, L)\right) \subset Q_{0}(K, L)$, $n>0$;

(iii) $\omega_{n}\left(Q_{n}(K, L)\right) \subset Q_{n}(K, L)$.

Hence these homomorphisms induce homomorphisms $h_{m, n}^{*}, \Delta_{n}^{*}$ and $\omega_{n}^{*}$ on the quotient rings $R_{n}(K, L), n \geqq 0$, and these induced homomorphisms satisfy formulae (7.6), (7.7) and (7.8). Thus, since each $\phi_{n}$ induces an isomorphism between $R_{n}(K, L)$ and $H(K \otimes L, n)$, the induced coefficient and Bockstein homomorphisms correspond to the coefficient and Bockstein homomorphisms in the cohomology spectrum of $K \otimes L$.

8. Homomorphisms induced by allowable cochain maps. In this section, the obvious definitions of homomorphisms induced by allowable cochain maps on the rings $S_{n}(K, L)$ are given. Let $K, K^{\prime}, L$ and $L^{\prime}$ be cochain complexes, and let $f: K \rightarrow K^{\prime}$ and $g: L \rightarrow L^{\prime}$ be allowable cochain homomorphisms. Then $\langle f, g\rangle_{0}: S_{0}(K, L) \rightarrow S_{0}\left(K^{\prime}, L^{\prime}\right)$ is defined by the formula

$$
\langle f, g\rangle_{0} \mid H(K, n) \otimes H(L, n)=f_{n} \otimes g_{n}
$$

where $f_{n}: H(K, n) \rightarrow H\left(K^{\prime}, n\right)$ and $g_{n}: H(L, n) \rightarrow H\left(L^{\prime}, n\right)$ are the homomorphisms induced by $f$ and $g$ respectively. Let $\langle f, g\rangle_{n}^{\prime}=\langle f, g\rangle_{0} \mid P_{n}(K, L)$. Then $\langle f, g\rangle_{n}: S_{n}(K, L) \rightarrow S_{n}\left(K^{\prime}, L^{\prime}\right)$ is defined by the formula

$$
\langle f, g\rangle_{n}=\left(\langle f, g\rangle_{n}^{\prime},\langle f, g\rangle_{n}^{\prime}\right) .
$$

Since $f$ and $g$ are allowable cochain homomorphisms, $f_{n}$ and $g_{n}$ commute with the corresponding Bockstein and coefficient homomorphisms (formula (2.9)). Thus $\langle f, g\rangle_{n}, n \geqq 0$ maps the generators of $Q_{n}(K, L)$ into the corresponding generators of $Q_{n}\left(K^{\prime}, L^{\prime}\right)$ and therefore $\langle f, g\rangle_{n}$ induces a homomorphism $\langle f, g\rangle_{n}^{*}: R_{n}(K, L) \rightarrow R_{n}\left(K^{\prime}, L^{\prime}\right)$.

The homomorphisms $\langle f, g\rangle_{n}$ and $\langle f, g\rangle_{n}^{*}, n \geqq 0$ satisfy the following functorial properties:

Lemma 8.1. (a) If $f: K \rightarrow K$ and $g: L \rightarrow L$ are identity homomorphisms, then $\langle f, g\rangle_{n}: S_{n}(K, L) \rightarrow S_{n}(K, L), n \geqq 0$, and $\langle f, g\rangle_{n}{ }^{*}: R_{n}(K, L) \rightarrow R_{n}(K, L)$ are identity homomorphisms. 
(b) If $f: K \rightarrow K^{\prime}, f^{\prime}: K^{\prime} \rightarrow K^{\prime \prime}, g: L \rightarrow L^{\prime}$ and $g^{\prime}: L^{\prime} \rightarrow L^{\prime \prime}$ are allowable homomorphisms, then

$$
\left\langle f^{\prime} f, g^{\prime} g\right\rangle_{n}=\left\langle f^{\prime}, g^{\prime}\right\rangle_{n}\langle f, g\rangle_{n}: S_{n}(K, L) \otimes S_{n}\left(K^{\prime \prime}, L^{\prime \prime}\right),
$$

and

$$
\left\langle f^{\prime} f, g^{\prime} g\right\rangle_{n}^{*}=\left\langle f^{\prime}, g^{\prime}\right\rangle_{n}^{*}\langle f, g\rangle_{n}^{*}: R_{n}(K, L) \rightarrow R_{n}\left(K^{\prime \prime}, L^{\prime \prime}\right) .
$$

These functorial properties follow from the corresponding properties for tensor products, direct sums and homology, the details of the verification being left to the reader.

The following theorem shows that $\phi_{n}, n \geqq 0$, is a natural homomorphism with respect to induced maps.

THEOREM 8.2. If $f: K \rightarrow K^{\prime}$ and $g: L \rightarrow L^{\prime}$ are allowable cochain homomorphisms, then

$$
\phi_{n}\langle f, g\rangle_{n}=(f \otimes g)_{n} \phi_{n}: S_{n}(K, L) \rightarrow H\left(K^{\prime} \otimes L^{\prime}, n\right)
$$

and

$$
\stackrel{*}{\phi}_{n}\langle f, g\rangle_{n}^{*}=(f \otimes g)_{n}{ }_{\phi_{n}}^{*}: R_{n}(K, L) \rightarrow H\left(K^{\prime} \otimes L^{\prime}, n\right) .
$$

This follows from the definitions, formula (2.9), and the fact that

$$
\alpha_{i}\left(f_{i} \otimes g_{i}\right)=(f \otimes g)_{i} \alpha_{i} .
$$

THEOREM 8.3. Let $f: K \rightarrow K^{\prime}$ and $g: L \rightarrow L^{\prime}$ be allowable cochain homomorphisms. Then $\langle f, g\rangle_{n}: S_{n}(K, L) \rightarrow S_{n}\left(K^{\prime}, L^{\prime}\right)$ preserves the ** products, i.e. $\langle f, g\rangle_{n}(x * * y)=\langle f, g\rangle_{n}(x) * *\langle f, g\rangle_{n}(y)$ for $x$ and $y$ in $S_{n}(K, L)$.

Proof. It suffices to prove this for the case listed in the definitions of the $* *$ products, e.g. formulae (4.12) and (6.7). The following formulae are used in this verification, where $x \in H(K, i) \otimes H(L, i), y \in H(K, j) \otimes H(L, j)$ and $u$ and $v$ are in $S_{0}(K, L)$.

$$
\begin{aligned}
\left(f_{i} \otimes g_{i}\right) h_{i, j}^{\prime} & =h_{i, j}^{\prime}\left(f_{j} \otimes g_{j}\right), \\
\langle f, g\rangle_{0}(u \cdot v) & =\langle f, g\rangle_{0}(u) \cdot\langle f, g\rangle_{0}(v), \\
D_{i}\left(f_{i} \otimes g_{i}\right) & =\left(f_{i} \otimes g_{i}\right) D_{i}, \\
\langle f, g\rangle_{0}\left(D_{i}(x) \cdot y\right) & =D_{i}\left(\langle f, g\rangle_{0}(x)\right) \cdot\langle f, g\rangle_{0}(y), \\
\langle f, g\rangle_{0}\left(x \cdot D_{j}(y)\right) & =\langle f, g\rangle_{0}(x) \cdot D_{j}\langle f, g\rangle_{0}(y), \\
\langle f, g\rangle_{n}^{\prime} & =\langle f, g\rangle_{0} \mid P_{n}(K, L) .
\end{aligned}
$$

TheOREM 8.4. If $f: K \rightarrow K^{\prime}$ and $g: L \rightarrow L^{\prime}$ are allowable cochain homomorphisms, then

$$
\langle f, g\rangle_{n} h_{n, m}=h_{n, m}\langle f, g\rangle_{m}: S_{m}(K, L) \rightarrow S_{n}\left(K^{\prime}, L^{\prime}\right),
$$




$$
\langle f, g\rangle_{0} \Delta_{n}=\Delta_{n}\langle f, g\rangle_{n}: S_{n}(K, L) \rightarrow S_{0}\left(K^{\prime}, L^{\prime}\right) .
$$

These formulae are consequences of the definitions. Their proofs are left to the reader.

Formulae (8.9) and (8.10) also hold for the homomorphisms $\langle f, g\rangle_{n}^{*}$, $h_{n, m}^{*}$ and $\Delta_{n}^{*}$ induced on the quotient rings $R_{n}(K, L)$.

The results of this section can be interpreted using the language of the theory of functors. (cf. Eilenberg and Steenrod [5, p. 111]).

For each integer $n \geqq 0, S_{n}$ is a function which assigns to each pair $(K, L)$ of cochain complexes a ring $S_{n}(K, L)$ and to each pair of allowable cochain homomorphisms $f: K \rightarrow K^{\prime}$ and $g: L \rightarrow L^{\prime}$, a ring homomorphism $\langle f, g\rangle_{n}: S_{n}(K, L)$ $\rightarrow S_{n}\left(K^{\prime}, L^{\prime}\right)$. Then Lemma 8.1 states that $S_{n}$ is a covariant functor on pairs of cochain complexes.

For each $n \geqq 0, R_{n}$ is a function which assigns, to each pair $(K, L)$ of finitely generated free cochain complexes, a ring $R_{n}(K, L)$, and to each pair of allowable cochain maps $f: K \rightarrow K^{\prime}$ and $g: L \rightarrow L^{\prime}$, a ring homomorphism $\langle f, g\rangle_{n}^{*}: R_{n}(K, L) \rightarrow R_{n}\left(K^{\prime}, L^{\prime}\right)$. From Lemma 8.1 , we see that $R_{n}$ is a covariant functor defined on pairs of finitely generated free cochain complexes.

9. Extension to infinite complexes and Čech cohomology. In this section, the functors $S_{n}$ and $R_{n}$ will be shown to commute with direct limits. This will then enable us to extend the results of the previous sections to infinite cochain complexes. We first list some known results about direct limits. Their proofs, in a general form, may be found in Cartan and Eilenberg [4].

(1) Direct limits commute with tensor products.

(2) Homology, as well as the coefficient and Bockstein homomorphisms, commute with direct limits.

(3) Direct sums commute with direct limits.

We shall use the notation of Eilenberg and Steenrod [5] for direct systems of groups.

Let the cochain complexes $\mathcal{K}$ and $\mathscr{L}$ be the direct limit of the direct system of cochain complexes $\{K, \pi\}$ and $\{L, \rho\}$ over the directed sets $M$ and $N$ respectively. The set $M \times N$ is directed by defining $(\alpha, \beta)<(\gamma, \delta)$ if $\alpha<\gamma$ and $\beta<\delta$ where $\alpha$ and $\gamma$ are in $M$ and $\beta$ and $\delta$ are in $N$. Let $\left\{S_{n}(K, L), S_{n}(\pi, \rho)\right\}$, $\left\{Q_{n}(K, L), Q_{n}(\pi, \rho)\right\}$ and $\left\{R_{n}(K, L), R_{n}(\pi, \rho)\right\}$ be the direct system of groups, subgroups and quotient groups defined as follows:

$$
\begin{array}{ll}
S_{n}(K, L)_{(\alpha, \beta)}=S_{n}\left(K_{\alpha}, L_{\beta}\right) ; & S_{n}(\pi, \rho)_{(\alpha, \beta)}^{(\gamma, \delta)}=S_{n}\left(\pi_{\alpha}^{\gamma}, \delta \beta\right. \\
\left.Q_{n}\right) ; \\
Q_{n}(K, L)_{(\alpha, o)}=Q_{n}\left(K_{\alpha}, L_{\beta}\right) ; & Q_{n}(\pi, \rho)_{(\alpha, \beta)}^{(\gamma \delta)}=S_{n}\left(\pi_{\alpha}^{\gamma}, \rho_{\beta}^{\delta}\right) \mid Q_{n}\left(K_{\alpha}, L_{\beta}\right) ; \\
R_{n}(K, L)_{(\alpha, \beta)}=R_{n}\left(K_{\alpha}, L_{\beta}\right) ; & R_{n}(\pi, \rho)_{(\alpha, \beta)}^{(\gamma, \delta)}=R_{n}\left(\pi_{\alpha}^{\gamma}, \rho_{\beta}^{\delta}\right) \text { for }(\alpha, \beta)<(\gamma, \delta) .
\end{array}
$$

THEOREM 9.1. $S_{n}(\mathcal{K}, \mathfrak{L}), Q_{n}(\mathcal{K}, \mathfrak{L})$ and $R_{n}(\mathcal{K}, \mathfrak{L})$ are the direct limits of the direct systems of groups, subgroups and quotient groups $\left\{S_{n}(K, L), S_{n}(\pi, \rho)\right\}$, $\left\{Q_{n}(K, L), Q_{n}(\pi, \rho)\right\}$ and $\left\{R_{n}(K, L), R_{n}(\pi, \rho)\right\}$ respectively. 
Proof. That $S_{n}(\mathscr{K}, \mathscr{L})$ is the direct limit of the direct system of groups $\left\{S_{n}(K, L), S_{n}(\pi, \rho)\right\}$ follows from the facts that tensor products, homology and direct sums commute with direct limits. To see that $Q_{n}(\mathcal{K}, \mathscr{L})$ is the direct limit of $\left\{Q_{n}(K, L), Q_{n}(\pi, \rho)\right\}$, one uses the fact that the homology spectrum commutes with direct limits. Thus the relations which define $Q_{n}(\mathcal{K}, \mathcal{L})$ can be pulled back to some $Q_{n}\left(K_{\alpha}, L_{\beta}\right)$. Since direct limits preserve exact sequences (cf. Eilenberg and Steenrod [5, p. 225]), $R_{n}(\mathcal{K}, \mathcal{L})$ s the direct limit of $\left\{R_{n}(K, L), R_{n}(\pi, \rho)\right\}$ because for each $\alpha$ in $M$ and $\beta$ in $N$, the following sequence is exact:

$$
0 \rightarrow Q_{n}\left(K_{\alpha}, L_{\beta}\right) \rightarrow S_{n}\left(K_{\alpha}, L_{\beta}\right) \rightarrow R_{n}\left(K_{\alpha}, L_{\beta}\right) \rightarrow 0 .
$$

THEOREM 9.2. If $K$ and $L$ are torsion free cochain complexes, then for each $i \geqq 0$,

(a) $\phi_{i}: S_{i}(K, L) \rightarrow H(K \otimes L, i)$ is a ring homomorphism onto,

(b) kernel $\phi_{i}=Q_{i}(K, L)$.

Proof. In $\S \S 4$ and 6 , we saw that $\phi_{i}$ is a ring homomorphism for $i \geqq 0$. Hence it suffices to prove that $\phi_{i}$ is onto and kernel $\phi_{i}=Q_{i}(K, L) . K$ is the union of its finitely generated allowable subgroups. Also for each pair $\left(K_{\alpha}, K_{\beta}\right)$ of finitely generated allowable subgroups, the subgroup generated by $K_{\alpha} \cup K_{\beta}$ is finitely generated and allowable. Hence $K$ is the direct limit of its finitely generated allowable subgroups (cf. Eilenberg and Steenrod [5, p. 229]). Similarly $L$ is the direct limit of its finitely generated allowable subgroups.

Since a finitely generated subgroup of a torsion free group is free, the following sequence is exact for any finitely generated allowable subgroups $K_{\alpha}$ of $K$ and $L_{\beta}$ of $L$ :

$$
0 \rightarrow Q_{i}\left(K_{\alpha}, L_{\beta}\right) \rightarrow S_{i}\left(K_{\alpha}, L_{\beta}\right) \rightarrow H\left(K_{\alpha} \otimes L_{\beta}, i\right) \rightarrow 0
$$

where the map $S_{i}\left(K_{\alpha}, L_{\beta}\right) \rightarrow H\left(K_{\alpha} \otimes L_{\beta}, i\right)$ is $\phi_{(\alpha, \beta)}$. Since direct limits preserve exact sequences, we conclude by Theorem 9.1 that the following sequence is exact:

$$
0 \rightarrow Q_{i}(K, L) \rightarrow S_{i}(K, L) \rightarrow H(K \otimes L, i) \rightarrow 0
$$

where the map $S_{i}(K, L) \rightarrow H(K \otimes L, i)$ is $\phi_{i}^{\infty}$. Since $\phi_{i}$ is natural, it commutes with the induced projections; hence $\phi_{i}^{\infty}=\phi_{i}$. This completes the proof of the theorem.

Corollary 9.3. If $K$ and $L$ are torsion free cochain complexes, then $\phi_{i}^{*}$ : $R_{i}(K, L) \rightarrow H(K \otimes L, i)$ is a ring isomorphism onto.

Let $X$ be a locally compact space, and let $H(X, i)$ be the Cech cohomology ring of $X$ with compact supports with the integers modulo $i$ as coefficients. It is known (cf. H. Cartan [3] or J. Leray [6]) that a torsion free cochain complex $K$ can be assigned to $X$ so that the cohomology ring $H(K, i)$ is naturally isomorphic to $H(X, i)$. It is also known that if $K$ and $L$ are torsion free cochain 
complexes assigned to the locally compact spaces $X$ and $Y$, as above, then the torsion free cochain complex $K \otimes L$ has the property that the cohomology ring $H(K \otimes L, i)$ is naturally isomorphic to $H(X \times Y, i)$ (for details and a proof see $[3$, p. 60] or $[6$, pp. 113-114]). Thus we see that the cohomology spectra of $X$ and $Y$ determine the cohomology rings $H(X \times Y, i)$. This is stated precisely in

Corollary 9.4. If $X$ and $Y$ are locally compact spaces, then $\phi_{i}^{*}: R_{i}(X, Y)$ $\rightarrow H(X \times Y, i)$ is a ring isomorphism onto.

Proof. Since the spectra of $X$ and $Y$ are isomorphic to the spectra of $K$ and $L$, where $K$ and $L$ are as above, we have $R_{i}(X, Y)$ is isomorphic to $R_{i}(K, L)$. By Corollary $9.3, R_{i}(K, L)$ is isomorphic to $H(K \otimes L, i)$ which in turn is isomorphic to $H(X \times Y, i)$.

Theorem 9.5. If $X$ and $Y$ are compact spaces, the Cech cohomology spectra of $X$ and $Y$ determine the Cech cohomology rings $H(X \times Y, i), i \geqq 0$.

Proof. The Cech cohomology ring, $H(X \times Y, i)$ is the direct limit of cohomology rings of nerves of finite coverings. One can verify that the set of "product" coverings $\left\{U_{i} \times V_{i}\right\}$ with $\left\{U_{i}\right\}$ a finite open covering of $X$ and $\left\{V_{j}\right\}$ a finite open covering of $Y$ is cofinal in the set of all coverings of $X \times Y$. Let $N(U)$ denote the nerve of a covering $U$. It can be verified that the simplicial product $([5$, pp. 66-70]) $N(U) \triangle N(V)=N(U \times V)$ where $U \times V$ is the product covering. Since $|N(U) \triangle N(V)|$ is homotopically equivalent to $|N(U) \times N(V)|$, it follows that $H(N(U) \times N(V))$ is naturally isomorphic to $H(N(U \times V))$. Hence

$$
\phi_{i}^{\infty}=\lim _{(U, V)} \phi_{i}^{(U, V)}
$$

is an isomorphism of $R_{i}(X, Y)$ onto $H(X \times Y, i)$ by Theorem 9.1.

Appendix. We shall prove Theorems 3.1 and 5.1 for the case where $K$ and $L$ are elementary complexes with two free generators. Let $K$ have free generators $a$ and $b$ in dimensions $p$ and $p+1$ respectively with $\delta a=t b, \delta b=0$, and let $L$ have free generators $a^{\prime}$ and $b^{\prime}$ in dimensions $q$ and $q+1$ respectively with $\delta a^{\prime}=s b^{\prime}, \delta b^{\prime}=0$. For easy reference, we list the cohomology groups of $K, L$ and $K \otimes L$ with the integers $\bmod n$ for coefficients, $n \geqq 0$. $Z_{n}[u]$ will denote a cyclic group of order $n$ with $u$ as a generator, $c=(n, t, s)$ and $d=(t, s)$.

$$
\begin{array}{rlrl}
H^{p}(K, n) & =Z_{(t, n)}\left[\left(\frac{n}{(n, t)} a\right)_{n}\right], & & n>0, \\
H^{p+1}(K, n) & =Z_{(t, n)}\left[b_{n}\right], & n \geqq 0, \\
H^{q}(L, n) & =Z_{(s, n)}\left[\left(\frac{n}{(n, s)} a^{\prime}\right)_{n}\right], & & n>0,
\end{array}
$$




$$
\begin{array}{rlrl}
H^{a+1}(L, n) & =Z_{(s, n)}\left[b_{n}^{\prime}\right], & & n \geqq 0, \\
H^{p+q}(K \otimes L, n) & =Z_{c}\left[\left(\frac{n}{c} a \otimes a^{\prime}\right)_{n}\right], & & n>0, \\
H^{p+q+1}(K \otimes L) & =Z_{d}\left[\left(\frac{1}{d} \delta\left(a \otimes a^{\prime}\right)\right)_{0}\right], & \\
H^{p+q+2}(K \otimes L, n) & =Z_{c}\left[\left(b \otimes b^{\prime}\right)_{n}\right], & & n \geqq 0, \\
H^{p+q+1}(K \otimes L, n) & =Z_{c}+Z_{c}, & & n>0 .
\end{array}
$$

It is convenient to describe this last group as the abelian group generated by

$$
E=\left(\frac{1}{d} \delta\left(a \otimes a^{\prime}\right)\right)_{n}, \quad G=(-1)^{p}\left(\frac{n}{(n, t)} a \otimes b^{\prime}\right)_{n}
$$

and

$$
F=\left(\frac{n}{(n, s)} b \otimes a^{\prime}\right)_{n}
$$

subject to the relations:

$$
\begin{aligned}
c E & =c F=c G, \\
\frac{n c}{(n, t)(n, s)} E & =\frac{t c}{(n, t) d} F+\frac{s c}{(n, s) d} G .
\end{aligned}
$$

All the other cohomology groups of $K, L$ and $K \otimes L$ are zero. The proof that the group generated by $E, F$ and $G$ subject to the above relations is the direct sum of two cyclic groups of order $c$ is left to the reader. Note that the integers $n c /[(n, t)(n, s)], t c /[(n, t) d]$ and $s c /[(n, s) d]$ are relatively prime in pairs.

To prove that $\phi_{n}$ is onto, it suffices to exhibit a pre-image for each generator. Thus we have

$$
\begin{array}{rlrl}
\phi_{n}\left(a_{c} \otimes a_{c}^{\prime}, 0\right) & =\left(\frac{n}{c} a \otimes a^{\prime}\right)_{n}, & & \\
\phi_{0}\left(a_{d} \otimes a_{d}^{\prime}\right) & =\left(\frac{1}{d} \delta\left(a \otimes a^{\prime}\right)\right)_{0}, & \\
\phi_{n}\left(b_{n} \otimes b_{n}^{\prime}, 0\right) & =\left(b \otimes b^{\prime}\right)_{n}, & \\
\phi_{0}\left(b_{0}^{\prime} \otimes b_{0}\right) & =\left(b \otimes b^{\prime}\right)_{0}, \\
\phi_{n}\left(b_{(n, s)} \otimes a_{(n, s)}^{\prime}, 0\right) & =F, \\
\phi_{n}\left((-1)^{p} a_{(n, t)} \otimes b_{(n, t)}^{\prime}, 0\right) & =G, \\
\phi_{n}\left(\alpha h_{c, d}^{\prime} D_{d}\left(a_{d} \otimes a_{d}^{\prime}\right), \beta a_{c} \otimes a_{c}^{\prime}\right) & =E,
\end{array}
$$


where $\alpha$ and $\beta$ are integers such that $\alpha n+\beta d=c$. The verification of the above formulae is left to the reader.

It is easy to see that $Q_{n}(K, L) \subset$ kernel $\phi_{n}, n \geqq 0$, because $\phi_{n}$ maps each generator of $Q_{n}(K, L)$ into zero. Thus to prove (b) kernel $\phi_{n}=Q_{n}(K, L)$, it suffices to prove $\left(\mathrm{b}^{\prime}\right)$ kernel $\phi_{n} \subset Q_{n}(K, L), n \geqq 0$.

In proving $\left(b^{\prime}\right)$, it will be convenient to introduce a graded structure in $S_{n}(K, L), n \geqq 0$. This is done for $S_{0}(K, L)$ by defining

$$
S_{0}^{r}(K, L)=\sum_{p+q=r} H^{p}(K) \otimes H^{q}(L)+\sum_{p+q=r-1} \sum_{n>0} H^{p}(K, n) \otimes H^{q}(L, n) .
$$

For $S_{n}(K, L)$, we define

$$
P_{n}^{r}(K, L)=\sum_{p+q-r} \sum_{i \mid n} H^{p}(K, i) \otimes H^{q}(L, i)
$$

and

$$
S_{n}^{r}(K, L)=P_{n}^{r}(K, L) \times P_{n}^{r-1}(K, L) .
$$

It is easily seen that $\phi_{n}\left(S_{n}^{r}(K, L)\right) \subset H^{r}(K \otimes L, n), n \geqq 0$. Thus to prove that kernel $\phi_{n} \subset Q_{n}(K, L), n \geqq 0$, it suffices to consider only homogeneous elements of $S_{n}(K, L)$.

An equivalence relation $\sim$ is introduced in $P_{n}(K, L)$ as follows: $u \sim v$ if $u-v$ is a linear combination of elements of the form $h_{\imath, j}(x) \otimes y-x \otimes h_{j, i}(y)$ where both $i$ and $j$ divide $n$. That $\sim$ is an equivalence relation follows immediately.

We now list some relations which are useful in the proof of $b^{\prime}$. We assume that $i$ divides $n$ and $c=(n, t, s)$.

$$
\begin{aligned}
\text { (A.1) } \quad h_{i, t}(x) \otimes h_{i, \ell}(y) & \sim \frac{i(n, t, s)}{(i, t)(i, s)} h_{c, t}(x) \otimes h_{c, s}(y), \\
\text { (A.2) } \quad \sum_{i \mid n} A_{i} h_{i, t}(u) \otimes h_{i, s}(v) & \sim\left(\sum_{i \mid n} A_{i} \frac{i(n, t, s)}{(i, t)(i, s)}\right)\left(h_{c, t}(u) \otimes h_{c, 8}(v)\right)
\end{aligned}
$$

where each $A_{i}$ is an integer.

$$
\begin{aligned}
h_{i, n}(x) \otimes h_{i, t}(y) & \sim \frac{(n, t)}{(i, t)} x \otimes h_{n, t}(y), \\
h_{i, t}(x) \otimes h_{i, n}(y) & \sim \frac{(n, t)}{(i, t)} h_{n, t}(x) \otimes y, \\
D_{i}(u) & \sim D_{n} h_{n, i}^{\prime}(u) \text { for } u \text { in } H(K, i) \otimes H(L, i) .
\end{aligned}
$$

Formulae (A.1), (A.3), (A.4) and (A.5) follow from formula (2.1), and the definition of $\sim$, while (A.2) follows from (A.1). 
An equivalence relation is introduced in $S_{n}(K, L)=P_{n}(K, L) \times P_{n}(K, L)$ as follows: For $u$ and $v$ in $S_{n}(K, L), u \equiv v\left(\bmod Q_{n}\right)$ if $u-v$ is in $Q_{n}(K, L)$. It is immediate that this is an equivalence relation.

For $x$ and $y$ in $P_{n}(K, L)$ the following three statements are equivalent:

(i) $x \sim y$,

(ii) $(0, x) \equiv(0, y) \bmod Q_{n}$,

(iii) $(x, 0) \equiv(y, 0) \bmod Q_{n}$.

This follows from the definitions of $Q_{n}(K, L)$ and the equivalence relations defined above.

The following equivalences modulo $Q_{n}$ are readily verified. A proof of (A.7) is given below. In each case $i$ divides $n$.

$$
\left(0, h_{i, 0}(x) \otimes h_{i, 0}(y)\right) \equiv 0 \bmod Q_{n},
$$

$$
\left(D_{i}(u), 0\right) \equiv\left(0, \frac{n}{i} u\right) \bmod Q_{n},
$$

$$
\left(0, D_{i}(u)\right) \equiv 0 \bmod Q_{n}
$$

for $u$ in $H(K, i) \otimes H(L, i), x$ in $H(K)$ and $y$ in $H(L)$.

Proof of (A. 7). It suffices to prove this for $u=x \otimes y$ in $H(K, i) \otimes H(L, i)$. $(n / i) x \otimes y=h_{i, n} h_{n, i}(x) \otimes y \sim h_{n, i}(x) \otimes h_{n, i}(y)$ by formula (2.1) and the definition of $\sim$. Hence $(0,(n / i) x \otimes y) \equiv\left(0, h_{n, i}(x) \otimes h_{n, i}^{\prime}(y)\right)$. By formula (A.5) and the relations defining $Q_{n}(K, L)$ this is $\equiv\left(D_{n} h_{n, i}^{\prime}(x \otimes y), 0\right) \equiv\left(D_{i}(x \otimes y), 0\right)$. The next two formulae are proved in a similar fashion.

$$
\begin{aligned}
\frac{d}{c}\left(\alpha h_{c, d}^{\prime} D_{d}(u), \beta h_{c, d}^{\prime} u\right) & \equiv\left(0, h_{c, d}^{\prime}(u)\right) \bmod Q_{n}, \\
\frac{n c}{(n, t)(n, s)} e & \equiv \frac{t c}{(n, t) d} f+\frac{s c}{(n, s) d} g \bmod Q_{n}
\end{aligned}
$$

where $d=(t, s), c=(n, t, s), u \in H(K, d) \otimes H(L, d), \alpha$ and $\beta$ are integers such that $\alpha n+\beta d=c, \quad e=\left(\alpha h_{c, d}^{\prime} D_{d}\left(h_{d, t}\left(x_{t}\right) \otimes h_{d, s}\left(y_{s}\right)\right), \quad \beta h_{c, t}\left(x_{t}\right) \otimes h_{c, s}\left(y_{s}\right)\right), f$ $=\left(h_{n, 0} \Delta_{t}\left(x_{t}\right) \otimes h_{n, s}\left(y_{s}\right), 0\right)$ and $g=\left(h_{n, t} \omega\left(x_{t}\right) \otimes h_{n, 0} \Delta_{s}\left(y_{s}\right), 0\right), x_{t} \in H(K, t)$ and $y_{s} \in H(L, s)$. The proof that kernel $\phi_{n} \subset Q_{n}(K, L)$ can be broken down into the following cases for $n>0$.

$$
\begin{array}{ll}
\text { Case (1) } & x \in S_{n}^{p+q+3}(K, L) . \\
\text { Case (2) } & x \in S_{n}^{p+q+2}(K, L) . \\
\text { Case (3) } & x \in S_{n}^{p+q+1}(K, L) . \\
\text { Case (4) } & x \in S_{n}^{p+q}(K, L) .
\end{array}
$$

We will only give proofs for Case (1), $n>0$, and Case (3), $n>0$ because the proofs for the other cases are similar.

Proof of Case 1, $n>0$. Let $x \in S_{n}^{p+q+3}(K, L), n>0$. Then $\phi_{n}(x)=0$ because 
$H^{p+q+8}(K \otimes L, n)=0$. But $x=\sum_{i \mid n} A_{i}\left(0, h_{i, 0}\left(b_{0}\right) \otimes h_{i, 0}\left(b_{0}^{\prime}\right)\right)$ which by formula (A.6) is $\equiv 0 \bmod Q_{n}$. Thus $x$ is in $Q_{n}(K, L)$.

Proof of Case $3, n>0$. Suppose $x \in S_{n}^{p+a+1}(K, L)$. Then by formulae (A.3), (A.4), (A.9), and (A.2), $x \equiv A e+B f+C g \bmod Q_{n}$ where $A, B$, and $C$ are integers, $c=(n, t, s), d=(t, s), \alpha$ and $\beta$ are integers such that $\alpha n+\beta d=c$,

$$
\begin{aligned}
& e=\left(\alpha h_{c, d}^{\prime} D_{d}\left(a_{d} \otimes a_{d}^{\prime}\right), \beta h_{c, t}\left(a_{\ell}\right) \otimes h_{c, s}\left(a_{s}^{\prime}\right)\right), \\
& f=\left(b_{n} \otimes h_{n, \ell}\left(a_{s}^{\prime}\right), 0\right)
\end{aligned}
$$

and

$$
g=\left(h_{n, t}\left(\omega\left(a_{t}\right)\right) \otimes b_{n}^{\prime}, 0\right) .
$$

Since $\phi_{n}(e)=E, \phi_{n}(f)=F$ and $\phi_{n}(g)=G, \phi_{n}(x)=0$ implies $A E+B F+C G=0$. Thus

$$
\begin{aligned}
A & \equiv m \frac{n c}{(n, t)(n, s)} \bmod c, \\
B & \equiv-m \frac{t c}{(n, t) d} \bmod c, \\
C & \equiv-m \frac{s c}{(n, s) d} \bmod c
\end{aligned}
$$

for some integer $m$. Since $c e=c f=c g$, this implies by formula (A.10) that $x \equiv 0 \bmod Q_{n}$.

One proceeds in a similar fashion in the (simpler) case $n=0$.

\section{BIBLIOGRAPHY}

1. M. Bockstein Universal systems of $\Delta$-homology rings C. R. (Doklady) Acad. Sci. URSS. vol. 37 (1942) pp. 243-245.

2. - Homological invariants of the topological product of two spaces, C. R. (Doklady) Acad. Sci. URSS. vol. 40 (1943) pp. 339-342.

3. H. Cartan, Seminaire de Topologie Algebrique ENS, III, 1950-1951.

4. H. Cartan and S. Eilenberg, Homological algebra, Princeton, 1956.

5. S. Eilenberg and N. Steenrod, Foundations of algebraic topology, Princeton, 1952.

6. J. Leray, L'anneau spectra et l'anneau filtre d'homologie d'une espace localement compact et d'une application continue, J. Math. Pures Appl. vol. 29 (1950) pp. 1-139.

7. H. Seifert and W. Threlfall, Lehrbuch der Topologie, Leipzig, 1934.

8. J. H. C. Whitehead, On simply-connected, four-dimensional polyhedra, Comment. Math. Helv. vol. 22 (1949) pp. 48-90.

BROWN UNIVERSITY

Providence, R.I.

UNIVERSITY OF MiChIGAN,

Ann Arbor, Mich. 